\title{
Is Modern Cosmology in Crisis?
}

Two well-regarded measurements for the expansion rate of the universe disagree, leaving cosmologists very puzzled. It may be that something large has been overlooked in our theory of the Big Bang. This discrepancy is called the Hubble tension and it has led to a very interesting conversation within the cosmology community.

Edwin Hubble is a legend in modern astronomy, often credited for discovering that the universe is expanding, and he deserves considerable credit for that observation. However, his work was not in a vacuum, and it built on earlier observations. For instance, it was Henrietta Leavitt who studied a type of star called the Cepheid variable. ${ }^{1}$

Cepheids change their brightness over time in regular intervals, and she discovered that there was a relationship between the length of time between dimming/brightening cycles and the star's intrinsic brightness. By exploiting this relationship, and measuring the star's apparent brightness, astronomers could determine the star's distance. This was an enormous advance in astronomy.

Vesto Slipher employed a spectroscope to study what were called at the time spiral nebulae (but what we now call spiral galaxies). He found that many of them had spectra that were shifted to the blue end of the spectrum. He concluded that this was due to the doppler effect and that these nebulae were preferentially moving away from Earth. ${ }^{1}$

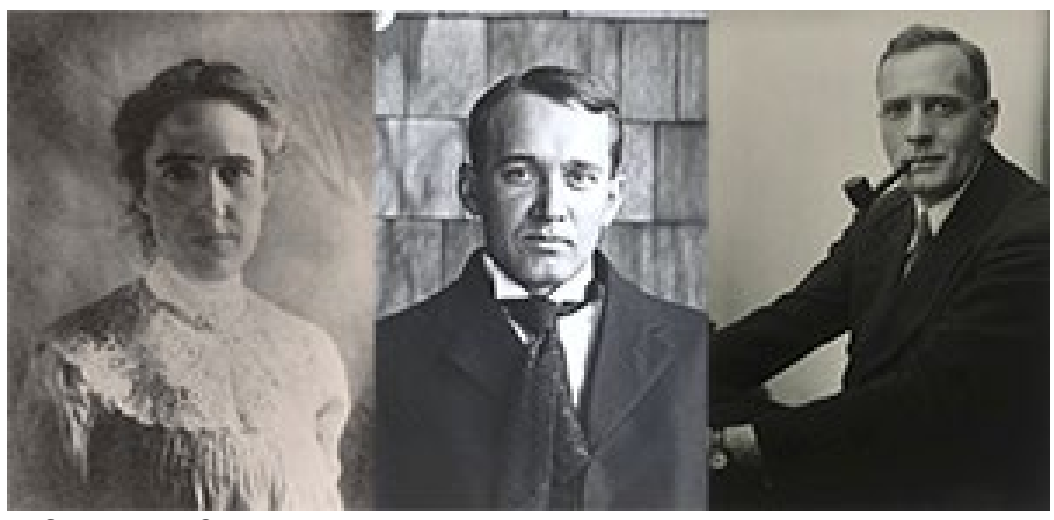

Figure 1: Early influential figures who contributed to the discovery that our universe is expanding: Henrietta Leavitt (left), Vesto Slipher (center), and Edwin Hubble (right). (Credit: Leavitt, Schlesinger Library, Radcliffe Institute, Harvard University; Slipher, Emilio Segrè Visual Archives; Hubble, Johan Hagemeyer).

Hubble's contribution was not either of these individual intellectual advances, but rather combining them. He found that there existed a relationship between the distance of a galaxy from Earth and its recessional velocity. The farther away a galaxy is from Earth, the faster it's moving compared to Earth's reference frame. Furthermore, Hubble was able to parametrize the relationship as a linear one. He found that the recessional velocity, $v_{r}$, is proportional to its distance, $D$, with a constant of proportionality of $H_{o}$. 


$$
v_{r}=H_{o} D
$$

The original constant of proportionality determined by Hubble was $500 \mathrm{~km} / \mathrm{s} / \mathrm{Mpc}$, where $1 \mathrm{Mpc}=1$ megaparsec $=3.26 \times 10^{6}$ light years. His original data are shown in Fig. 2.

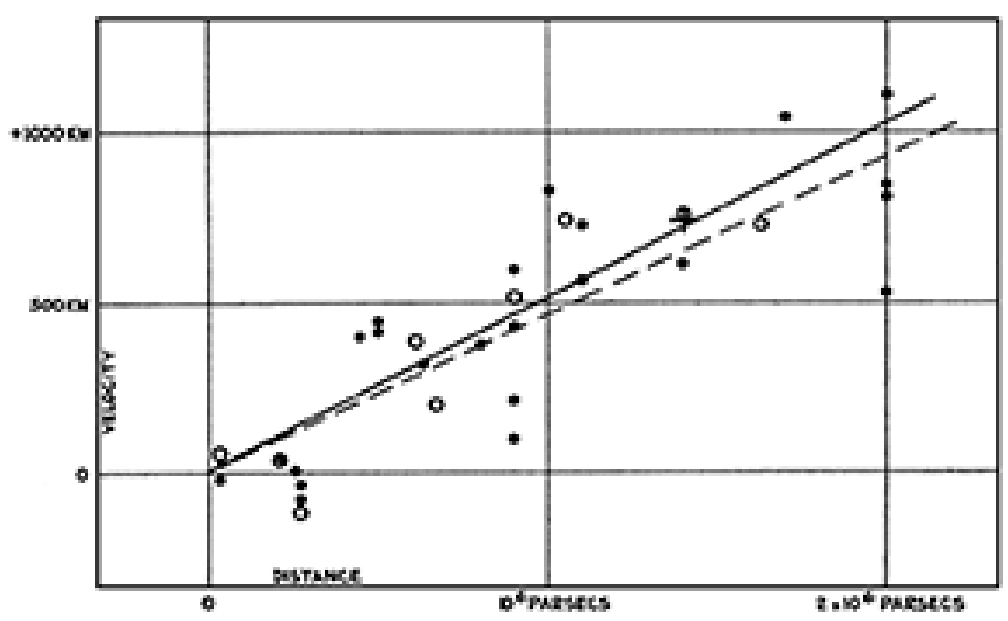

Figure 2: Hubble's original data. Each dot is a galaxy. The slope of the line is approximately $500 \mathrm{~km} / \mathrm{s} / \mathrm{Mpc}$ and there is some scatter in the data. (Credit: From Ref. 2).

Hubble's original paper was published in 1929 and extended to a distance of about 2 Mpc. At the time, this distance was approximately the maximum range over which the use of Cepheid variables is a good approach to measure distances. For more distant galaxies, astronomers have turned to Type la supernovae, which is a class of supernovae for which the intrinsic brightness can be well determined.

Figure 3 shows a modern version of Hubble's original graph, out to a distance of approximately $700 \mathrm{Mpc}$. Hubble's original observation of the linear correlation between distance and recessional velocity continues to hold.

Increases in the precision of the measurement and a much-increased lever arm has led to a large reduction in the estimate of the Hubble constant, accompanied by a comparable reduction in the uncertainty. A modern estimate for the Hubble constant is $H_{0} \sim 70 \mathrm{~km} / \mathrm{s} / \mathrm{Mpc}$. Figure 4 shows the historical improvement in estimates of this parameter. 


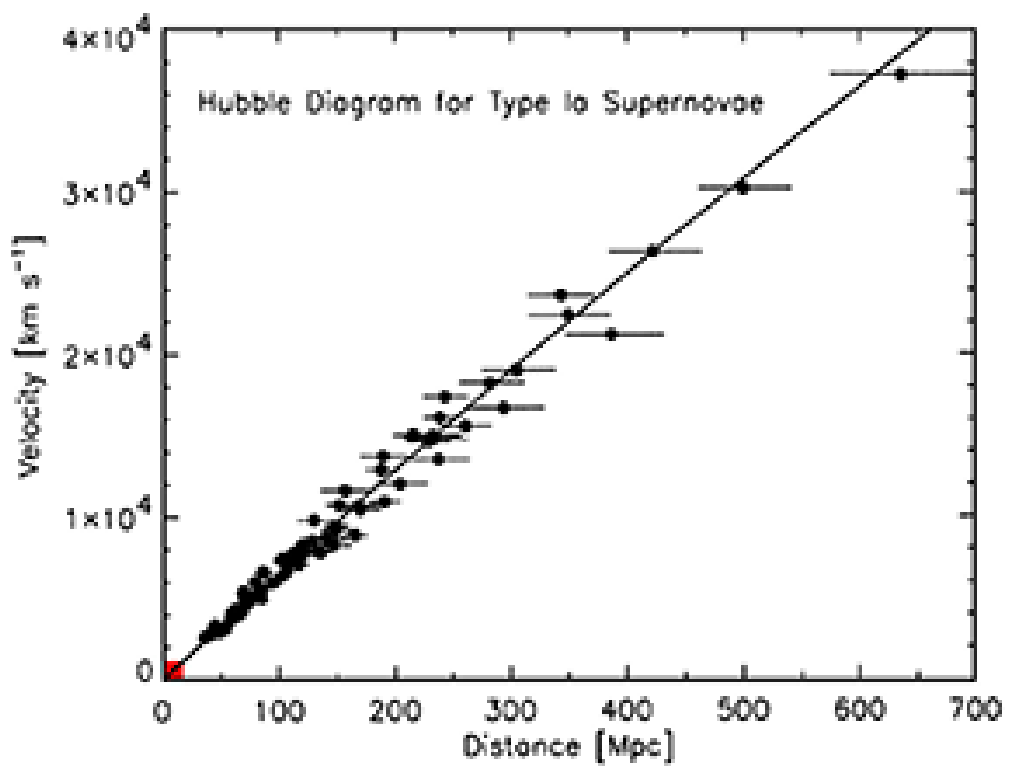

Figure 3: A modern equivalent of Fig. 2, using Type la supernovae to extend the initial measurement to larger distances. The small red square in the lower left corner of the figure signifies the data range originally explored by Hubble. (Credit: From Ref. 3).

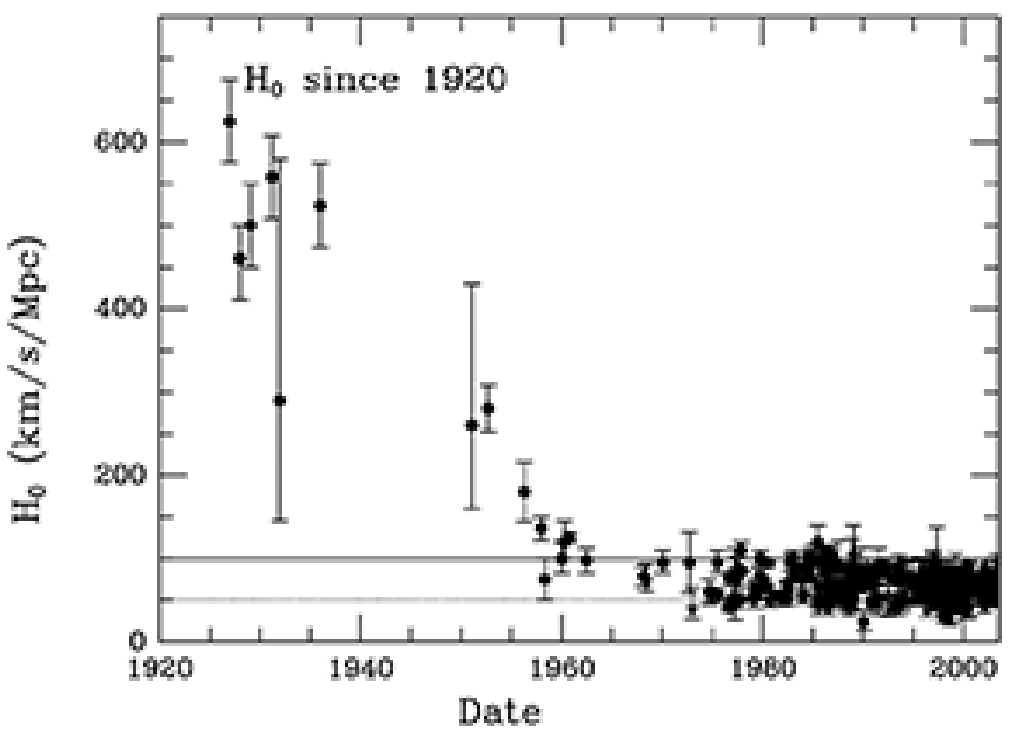

Figure 4: Estimates of the Hubble parameter have improved in both accuracy and precision since the initial estimate. Modern measurements are closer to the ballpark of 70 km/s/Mpc. (Credit: From Ref. 1). 


\section{An Inconstant Constant}

When is a constant not constant? This isn't a trick question, and the answer is obvious. It's not constant when it changes. And, if you think about it for a minute, the Hubble constant is misnamed.

The universe began in a singular event that we colloquially call the Big Bang. ${ }^{4}$ While the particulars of the Big Bang theory are often misunderstood, the broad strokes are clear. The visible universe was once much smaller and, shortly after the universe began, it was expanding very quickly. Then, over the eons, the gravitational attraction of the matter of the universe caused the expansion of the universe to slow down. Conceptually, this is no different than the velocity of a ball thrown upward from the surface of Earth.

The expansion rate slowed for the first nine billion years of the life of the universe. Then, about five billion years ago, a repulsive form of gravity, colloquially called "dark energy," became dominant and the expansion of the universe speeded up. ${ }^{5}$ It continues to speed up today.

The bottom line is that, on cosmic timescales, the expansion rate of the universe changed. It wasn't constant. Thus, the term "the Hubble constant" is misleading, or at least incomplete. The Hubble constant is actually the expansion rate of the universe as a function of distance in the current era.

\section{Indirect Determination of Hubble Constant}

Over the past century, astronomers have constantly refined their model of the universe. The two largest advances in accurately describing the cosmos are the theory of gravity at large scales (e.g., Einstein's theory of general relativity) and identifying the important mass and energy components of the universe. These components are: (1) ordinary matter, ultimately composed of protons, neutrons, and electrons; (2) dark matter, which is a form of matter that interacts gravitationally, but not according to the other known forces $^{6}$; and (3) dark energy, which is a repulsive form of gravity. These three components have been measured to be: (4.9\%, ordinary matter), $(26.8 \%$, dark matter), and $\left(68.3 \%\right.$, dark energy) ${ }^{7}$

The methodology for determining the energy content of the universe is an indirect one, involving data from the early universe that researchers fit to a model that includes both the Big Bang and the universe as a whole. This model is called the Cosmic Concordance model, and it includes every known and relevant astronomical phenomenon, including ordinary matter, dark matter, dark energy, the cosmic microwave background (CMB), structure in the distribution of galaxies at cosmological distance scales, etc. The Cosmic Concordance model is also sometimes called the $\Lambda$ CDM model, where $\Lambda$ is a parameter of dark energy and CDM is an acronym for "cold dark matter." 
The fitting procedure is rather complex, ${ }^{7}$ as it includes an enormous amount of data, but the phenomenon that has the largest impact on the fit is the CMB. The CMB was discovered in 1964 by Arno Penzias and Bob Wilson and is a radio signal that is the remnant of the hot phase of the Big Bang. The initial measurement showed that the spectrum of the CMB was shockingly uniform across the cosmos and was consistent with being a black body radiator with a temperature of $2.726 \mathrm{~K}$.

In 1998, the COBE collaboration discovered that the CMB had tiny non-uniformities, varying from the mean by about one part in $10^{4}$. The CMB actually had a subtle pattern of hotter and colder spots. Two follow-on experiments, called WMAP and Planck both confirmed the COBE observation and improved the precision. Figure 5 shows the temperature variation of the $\mathrm{CMB}$.

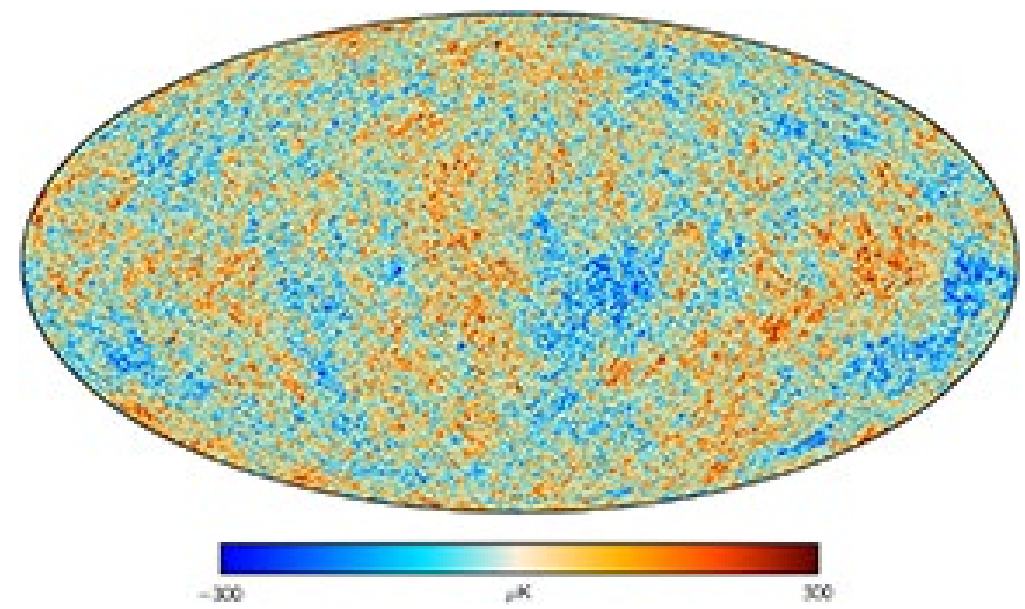

Figure 5: Temperature map of the whole sky as measured by the Planck collaboration. The red and blue spots indicate a maximum $300 \mu \mathrm{K}$ variation from the mean. (Credit: ESA/Planck) [End caption]

The pattern of hot and cold spots contains within it a substantial amount of information. Researchers can fit the pattern to a series of orthogonal functions, called spherical harmonics. This is conceptually identical to the Fourier analysis approach of representing any cyclical function as a weighted series of sine and cosine functions. The methodology is described in Ref. 8.

Figure 6 shows the impact of the different spherical harmonics from order 1 to 2508 . The dominant multipole is around $n=200$, which roughly corresponds to variations in the temperature of the $\mathrm{CMB}$ with an angular scale of order one degree. The curve is a fit to the data when the matter and energy components of the universe, the Hubble constant, and other parameters are allowed to vary. NASA has an online tool that allows someone to investigate the sensitivity of the fit to changes in the parameters. ${ }^{9}$ 


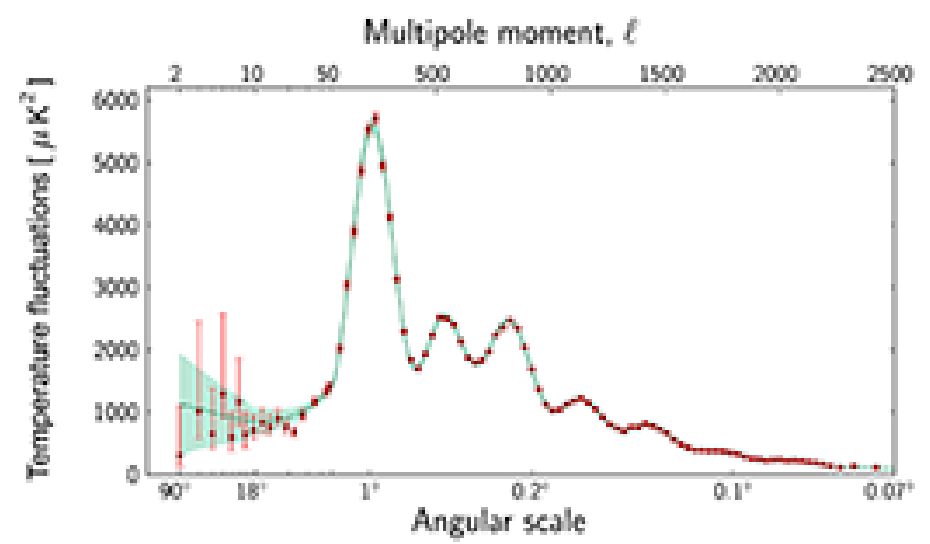

Figure 6: Distribution of multipole components of the CMB background radiation when represented as a series of spherical harmonics. The most common variation has an angular size of about one degree. The curve is a fit to the data. (Credit: ESA/Planck) [End caption]

By fitting the data in Fig. 6, and other similar distributions, one outcome is a precise estimate of the Hubble constant. However, this estimate disagrees with direct measurements.

\section{The Hubble Tension}

The Hubble parameter, measured directly via distance estimates using Cepheid variables and Type la supernovae, is estimated to be about $74 \mathrm{~km} / \mathrm{s} / \mathrm{Mpc}$. In contrast, the same number estimated by the Planck collaboration from CMB data is about 67 $\mathrm{km} / \mathrm{s} / \mathrm{Mpc}$.

One's initial reaction is that these numbers are in reasonable agreement. After all, the difference is about $10 \%$. However, like all measurements, a proper interpretation requires that one know the uncertainty. While this discrepancy has been known for some time, uncertainties were such that it was possible to dismiss them as not being statistically significant. This changed in 2019.

In 2018, the Planck collaboration released an encyclopedic description of their scientific results, including an estimate of the Hubble constant. ${ }^{7}$ Their result is $H_{o}=67.4 \pm 0.5$ $\mathrm{km} / \mathrm{s} / \mathrm{Mpc}$.

A group of researchers led by Adam Riess ${ }^{10}$ measured the Hubble constant directly, with a value of $H_{0}=74.03 \pm 1.42 \mathrm{~km} / \mathrm{s} / \mathrm{Mpc}$. This result differs by $6.6 \mathrm{~km} / \mathrm{s} / \mathrm{Mpc}$ and is discrepant with a significance of 4.4 standard deviations, which is $0.001 \%$ likely to be a statistical fluctuation.

The Riess measurement improved over earlier estimates because they conducted a precise study of the Cepheid variables in the Large Magellanic Cloud, which is exactly the approach followed by Henrietta Leavitt a century ago. 


\section{Possible Explanations}

Both measurements are well-regarded by the scientific community, performed by excellent scientists. Whenever two good measurements disagree by an amount that is statistically significant, it opens the question, "What is going on?"

Taking a step back, we should review the situation. The direct measurement (Riess and similar efforts) is predicated on an estimate of the distance of cosmological objects out to $700 \mathrm{Mps}$ away. The weakness of this particular measurement is an overlooked bias in the methodology whereby distances are determined. This is an entirely credible possible explanation of the disagreement, as astronomical distance measurements are notoriously difficult.

The indirect measurement (Planck et al.) depends on both measurements of the angular distribution of temperature differences (which is very precise) and a model whereby the observed temperature differences (which probe a time about 380,000 years after the universe began) are propagated via the Cosmic Concordance model to modern days (13.8 billion years later). Here the dominant weakness is whether the model accurately includes all effects.

Certainly, the more exciting prospect is that the Cosmic Concordance model is incomplete. If so, new physical phenomena will be required to extend it.

One such possibility was proposed by postdoctoral research Vivian Poulin and professor Marc Komionkowski at Johns Hopkins and Tristan Smith at Swarthmore. ${ }^{11}$ In this paper, they postulated another period of inflation that is not part of accepted theory.

In the Cosmic Concordance model, the universe experienced a period of superluminal expansion called inflation during the time period of about $10^{-36}$ to $10^{-32} \mathrm{~s}$ after the beginning of the universe. This expansion was driven by a scalar field of energy that switched off after driving the expansion. Then, about five billion years ago, another energy field that we call dark energy began to dominate the universe, causing the expansion of the universe to accelerate. In between, the expansion of the universe was ballistic, driven by the initial expansion and slowed by conventional gravity.

Poulin and collaborators solve the discrepancy by proposing a third period of accelerated expansion in a timeframe close to when the CMB became visible. This accelerated expansion would have existed only for a brief period of time and accounted for the $10 \%$ discrepancy. Another paper by Lisa Randall and collaborators presented a similar strategy. ${ }^{11}$

Other proposed explanations are difficult to reconcile with data. Astronomers have observed late-universe galaxy cluster structures, which set limits on many possible proposed additions to the Concordance Mode. In addition, observed baryon acoustic oscillations (BAOs) greatly limits new ideas. ${ }^{11,12}$ (BAOs are the recurring pattern of 
clusters of galaxies and voids seen in the universe. They can roughly be thought of as soap bubbles in the pattern of matter distributed throughout the cosmos. The "size" of the bubbles sets severe constraints on proposed new physics.)

A more prosaic explanation of the Hubble tension has also been advanced. It is possible that the Hubble tension arises not due to new physics, but rather reflects an unknown systematic in the measurement. Riess's group's measurement ${ }^{10}$ has been confirmed by other measurements, for example one with the imaginative name HOLICOW, using distant quasars. ${ }^{13}$ However, not all groups agree.

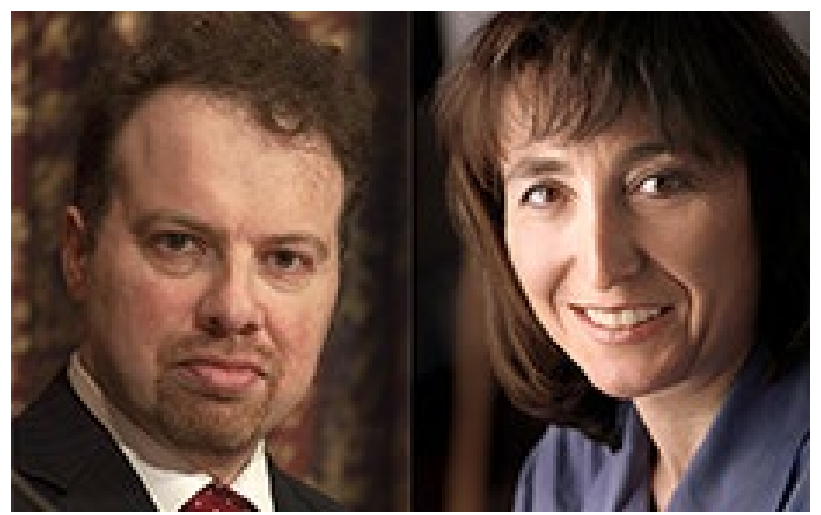

Figure 7: Adam Riess (left) and Wendy Freedman (right) are two leading scientists who are debating possible origins for the Hubble tension. (Credit: Stockholm University/Holger Motzkau [Riess] and The Gruber Foundation [Freedman]).

A group helmed by Wendy Freedman of the University of Chicago is pursuing another method to determine cosmic distances. She and her collaborators are using red giant stars in their work. Stars like our Sun eventually use all of their hydrogen and then switch over to burning helium. When they do so, they inflate to red giants, with a known luminosity. Using red giants, Freedman's group determined ${ }^{14}$ a value of the Hubble constant of $69.8 \pm 0.8$ (stat) \pm 1.7 (sys) $\mathrm{km} / \mathrm{s} / \mathrm{Mpc}$, about halfway between the earlier direct and indirect measurements.

While this measurement could prove to be problematic to claims that a tension exists in measurements of the Hubble constant, Riess and collaborators have already responded with criticisms of the Freedman methodology. ${ }^{15}$ They claim that Freedman and collaborators did not properly take into account dust in the Large Magellanic Cloud. And the conversation continues.

\section{Denouement}

So, what will the outcome be? Of course, nobody knows. It is certainly possible that the Hubble tension is the first indication of a missing piece of the Cosmic Concordance model. However, determining the distance of faraway objects in the universe is a very difficult thing. It is quite possible that the discrepancy is simply an artifact of a systematic effect that has not been properly take into account. Either way, the debate is an exciting one and we will have to wait to find out what the answer is. 


\section{References}

[1] R. P. Kirshner, "Hubble's diagram and cosmic expansion," Proc. Natl. Acad. Sci. 101 (1), 8-13 (2004).

[2] E. P. Hubble, "A relation between distance and radial velocity among extra-galactic nebulae," Proc. Natl. Acad. Sci. 15, 168-173 (1929), https://www.pnas.org/content/15/3/168?ijkey=2b9b8fc2b5f3f95f1fb3b54e2b4c700b5 2fa14e2\&keytype2=tf ipsecsha

[3] S. Jha, Ph.D. thesis (Harvard University, Cambridge, MA). (Author: title and date?)

[4] D. Hooper, At the Edge of Time: Exploring the Mysteries of our Universe's First Seconds (Princeton University Press, 2019).

[5] D. Lincoln and B. Nord, "The expanding universe: Dark energy," Phys. Teach. 52, 337 (Sept. 2014).

[6] D. Lincoln, “Dark matter,” Phys. Teach. 51, 134 (March 2013).

[7] Planck Collaboration, "Planck 2018 results. VI. Cosmological parameters," arXiv:1807.06209, https://arxiv.org/abs/1807.06209; https://sci.esa.int/web/planck/151557-planck-new-cosmic-recipe.

[8] Planck Collaboration, "Planck 2018 results. VII. Isotropy and statistics of the CMB," arXiv:1906.02552; M. Tanabashi et al. (Particle Data Group), "Review of particle physics," Phys. Rev. D 98, 030001 (2018) and 2019 update.

[9] "Build a Universe," Wilkinson Microwave Anisotropy Probe, NASA.gov, https://map.gsfc.nasa.gov/resources/camb_tool/index.html.

[10] A. G. Riess, S. Casertano, W. Yuan, L. M. Macri, and D. Scolnic, "Large Magellanic Cloud Cepheid standards provide a $1 \%$ foundation for the determination of the Hubble constant and stronger evidence for physics beyond $\Lambda$ CDM," Astrophys. J. 876, 85 (2019).

[11] Poulin, V., Early Dark Energy can Resolve the Hubble Tension, Phys. Rev. Lett. 122, 221301 (2019); arXiv:1811.04083; Agrawal, P., Rock 'n' Roll Solutions to the Hubble Tension, arXiv:1904.01016v1.

[12] Eisenstein, D. J., Dark energy and cosmic sound, New Astronomy Reviews. 49 (79): 360 (2005). Bibcode: 2005ASPC..339..187E

[13] Wong, K. C. et al., HOLiCOW XIII. A 2.4\% measurement of HO from lensed quasars: $5.3 \sigma$ tension between early and late-Universe probes, arXiv:1987.04869.

[14] Freedman, W. L., et al. 2019, ApJ,882, 34, doi: 10.3847/1538-4357/ab2f7

[15] Yuan, W et al., Consistent Calibration of the Tip of the Red Giant Branch in the Large Magellanic Cloud on the Hubble Space Telescope Photometric System and a Re-determination of the Hubble Constant, arXiv:1908.00993v3. 